\begin{tabular}{|c|l|}
\hline Title & Metal-induced gap states in epitaxial organic-insulator/metal interfaces \\
\hline Author(s) & $\begin{array}{l}\text { Kiguchi, Manabu; A rita, Ry otaro; Y oshikawa, Genki; Tanida, Y oshiaki; I keda, Susumu; Entani, Shiro; Nakai, Ikuyo; } \\
\text { Kondoh, Hiroshi; Ohta, Toshiaki; Saiki, Koichiro; A oki, Hideo }\end{array}$ \\
\hline Citation & $\begin{array}{l}\text { Physical Review B, 72(7), 075446 } \\
\text { https://doi.org/10.1103/PhysRevB.72.075446 }\end{array}$ \\
\hline Issue Date & 2005-08 \\
\hline Doc URL & http://hdl.handle.net/2115/29745 \\
\hline Rights & Copyright $\odot$ 2005 A merican Physical Society \\
\hline Type & article \\
\hline File Information & PRB72-07.pdf \\
\hline
\end{tabular}

Instructions for use 


\title{
Metal-induced gap states in epitaxial organic-insulator/metal interfaces
}

\author{
Manabu Kiguchi, ${ }^{1, *}$ Ryotaro Arita, ${ }^{2, \dagger}$ Genki Yoshikawa, ${ }^{3}$ Yoshiaki Tanida, ${ }^{4}$ Susumu Ikeda, ${ }^{1}$ Shiro Entani, ${ }^{1}$ Ikuyo Nakai, ${ }^{3}$ \\ Hiroshi Kondoh, ${ }^{3}$ Toshiaki Ohta, ${ }^{3}$ Koichiro Saiki, ${ }^{1,3}$ and Hideo Aoki ${ }^{2}$ \\ ${ }^{1}$ Department of Complexity Science and Engineering, University of Tokyo, Kashiwa, Chiba 277-8561, Japan \\ ${ }^{2}$ Department of Physics, University of Tokyo, Hongo, Tokyo 113-0033, Japan \\ ${ }^{3}$ Department of Chemistry, University of Tokyo, Hongo, Tokyo 113-0033, Japan \\ ${ }^{4}$ Fujitsu Laboratories Ltd., Atsugi, Kanagawa 243-0197, Japan
}

(Received 11 January 2005; revised manuscript received 2 June 2005; published 31 August 2005)

\begin{abstract}
We have shown, both experimentally and theoretically, that the metal-induced gap states (MIGS) can exist in epitaxially grown organic insulator/metal interfaces. The experiment is done for alkane/Cu(001) with an element-selective near edge x-ray absorption fine structure (NEXAFS), which exhibits a prepeak indicative of MIGS. An $a b$ initio electronic structure calculation supports the existence of the MIGS. When the Cu substrate is replaced with $\mathrm{Ni}$, an interface magnetism may be possible with a carrier doping.
\end{abstract}

DOI: 10.1103/PhysRevB.72.075446

PACS number(s): 73.20.-r, 71.15.Mb, 73.40.Ns

\section{INTRODUCTION}

While there are mounting interests in the nature of heterointerfaces, organic crystal/metal interfaces are especially intriguing due to their diverse possibilities not found in inorganic counterparts, which may facilitate in controlling and designing properties of interfaces. Electronic structures of organic/metal interfaces are important from a technological point of view as well, since the performance of devices should strongly depend on the electronic structure at the interface.

Recent years have in fact witnessed several interesting results on organic-crystal/metal interfaces. ${ }^{1}$ One crucial factor in such organic-insulator/inorganic-metal heterointerfaces is the energy level alignment at the interface, which is still far from being fully understood. The band alignment, measured by ultraviolet photoelectron spectroscopy (UPS) and Kelvin probe, has been discussed in terms of various effects, such as electron transfer, image effect, modification of the surface dipole at metal surface, chemical interaction and interfacial states, etc. ${ }^{2}$ However, there is no generally accepted picture for organic/metal interfaces, which is contrasted with inorganic-semiconductor/metal interfaces where the band alignment is known to be governed by the interface states called the metal-induced gap states (MIGS). ${ }^{3}$ So a study of MIGS at the organic/metal interfaces should be imperative.

The notion of MIGS was first introduced for semiconductor/metal junctions in discussing the Schottky barrier. ${ }^{3}$ MIGS are roughly free-electron-like metal wave functions penetrating into the semiconducting side, where the penetration depth is inversely proportional to the band gap in the conventional band picture. So a usual wisdom dictates that MIGS would be far too thin in insulator/metal interfaces to be relevant. The present authors succeeded in fabricating epitaxial alkali-halide/metal interfaces, which has enabled us to obtain unambiguous evidences especially from the near edge $\mathrm{x}$-ray absorption fine structure (NEXAFS) that MIGS are in fact formed at the inorganic-insulator/metal interfaces. $^{4-6}$ One reason for the successful detection of MIGS is the following: while signals from the interface are obscured by the signal from the substrate in conventional experimental methods, because the signals in these methods involve significant contributions from the substrate, NEXAFS, based on the $\mathrm{x}$-ray absorption of the atom, provides information on the alkali halide with influences from the substrate being negligible.

While alkali halides are typical inorganic insulators, interface states for organic-insulator/metal interfaces are a totally different issue, since chemical bonds in organic insulators are covalent as opposed to ionic bonds in inorganic, ionic insulators, and it is a fundamental question to ask whether the formation of MIGS at insulator/metal interfaces are universal enough to accommodate organic insulators. Experimentally, interface states in atomically well-defined organic-insulator/ metal interfaces have yet to be observed to our knowledge ${ }^{7}$ despite their prime importance. One obvious reason for this is difficulties in fabricating atomically well-defined organicinsulator/metal interfaces and in detecting signals from the interface. ${ }^{4}$ However, recent developments in the molecular beam epitaxy (MBE) technique have made it possible to prepare various types of heterointerfaces. ${ }^{8,9}$ Some organic films are in fact begun to be epitxially grown on metal substrates in a layer-by-layer fashion with MBE. ${ }^{10}$

Given this background, the purpose of the present paper is to examine the interface states in an atomically well-defined organic insulator (an alkane; $\mathrm{C}_{44} \mathrm{H}_{90}$ ) grown on $\mathrm{Cu}$ substrate. We have found, with the element-selective NEXAFS, a prepeak indicative of metal-induced gap states. At the same time we have performed an $a b$ initio electronic structure calculation. The theoretical result shows that (i) MIGS do exist at the organic insulator/metal interface, and (ii) when we replace $\mathrm{Cu}$ with $\mathrm{Ni}$, in which narrow $3 d$ bands rather than wide $4 s$ bands dominate the electronic properties around the Fermi energy, an interface magnetism is predicted to be possible with a carrier doping.

\section{EXPERIMENT}

The experiments were performed with a UHV chamber at the soft $\mathrm{x}$-ray beam line BL-7A in the Photon Factory in the Institute of Materials Structure Science, Japan. We have em- 


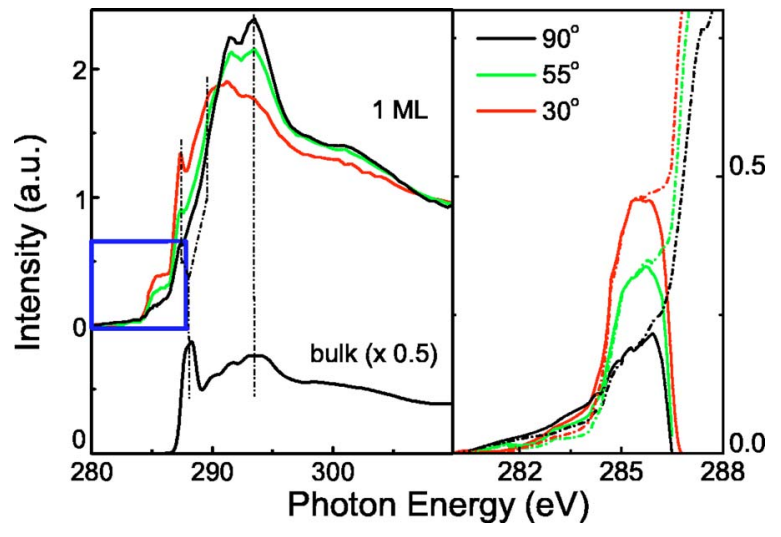

FIG. 1. (Color online) Experimental result for the C $K$-edge NEXAFS spectra in $1 \mathrm{ML} \mathrm{C}_{44} \mathrm{H}_{90}$ (TTC) films grown on $\mathrm{Cu}(001)$ for the $\mathrm{x}$-ray incidence angle $\theta$ varied over $30^{\circ}, 55^{\circ}, 90^{\circ}$. We also display for comparison the result for a multilayer TTC (bulk) on $\mathrm{Cu}(001)$ in black (which is almost $\theta$ independent, here for $\theta=90^{\circ}$ ). All the spectra are normalized by their edge jump. Right-hand panel is a blowup of the prepeaks, obtained by subtracting the bulk (multilayer) spectrum which has no structures at the edge onset.

ployed an alkane $\left(\mathrm{C}_{n} \mathrm{H}_{2 n+2}\right.$ with $n=44$; tetratetracontane or TTC) as a simplest hydrocarbon, where the properties of this series of molecules are known to be similar except for a variation in the band gap with $n$. A clean $\mathrm{Cu}(001)$ surface was prepared by repeated cycles of Ar sputtering and annealing at $900 \mathrm{~K}$. TTC was then evaporated on $\mathrm{Cu}(001)$ with the substrate temperature of $300 \mathrm{~K}$ with a Knudsen cell. Realtime observation of crystallinity and orientation of the film was probed by reflection high energy electron diffraction (RHEED) with a microchannel plate. Half-order streaks appeared only along the [110] azimuth direction for a 1 monolayer (ML) thick $\mathrm{TTC} / \mathrm{Cu}(001)$. This indicates that the TTC film epitaxially grows on $\mathrm{Cu}(001)$ with its molecular axis parallel to [110] azimuth of the $\mathrm{Cu}$ substrates in a layer-bylayer fashion. ${ }^{10}$ Carbon $K$-edge NEXAFS spectra were then obtained in-situ by the partial electron yield method with a microchannel plate. Since electrons with energies about $200 \mathrm{eV}$ are detected, the probing depth is estimated to be $0.8 \mathrm{~nm}$, which is deep enough to study the electronic structure for the flat-lying $1 \mathrm{ML}$ thick TTC film. Energy resolution was $0.3 \mathrm{eV}$ at $\mathrm{C} K$-edge. The NEXAFS spectra were taken at $30^{\circ}, 55^{\circ}, 90^{\circ} \mathrm{x}$-ray incident angles from the surface parallel. We can qualitatively know the anisotropy of the orbital by changing the incident angles. ${ }^{11}$

Figure 1 shows the NEXAFS for a 1 ML TTC on $\mathrm{Cu}(001)$, as compared with the result for a bulk (multilayer) TTC film. In $\mathrm{C} K$-edge NEXAFS for $n$-alkane, there are many (at least five) resonances, and all the resonances have not been assigned up to now. ${ }^{12}$ So we only discuss the assigned $\sigma^{*}(\mathrm{C}-\mathrm{C})$ and $\sigma^{*}(\mathrm{C}-\mathrm{H})$ resonances. For a bulk TTC, a broad peak at about $293 \mathrm{eV}$ is assigned to a $\mathrm{C} 1 s \rightarrow \sigma^{*}(\mathrm{C}$ C) resonance, while the peak at $288 \mathrm{eV}$ to a C $1 s \rightarrow \sigma^{*}(\mathrm{C}$ $\mathrm{H}) .{ }^{12}$ The $\sigma^{*}(\mathrm{C}-\mathrm{C})$ peak whose transition moment is parallel to the $\mathrm{C}-\mathrm{C}-\mathrm{C}$ plane is most enhanced at the normal $\mathrm{x}$-ray incidence, which confirms that the TTC grows on $\mathrm{Cu}(001)$ with its molecular axis parallel to the substrate. On the other hand, the $\sigma^{*}(\mathrm{C}-\mathrm{H})$ peak, whose transition moment perpen-
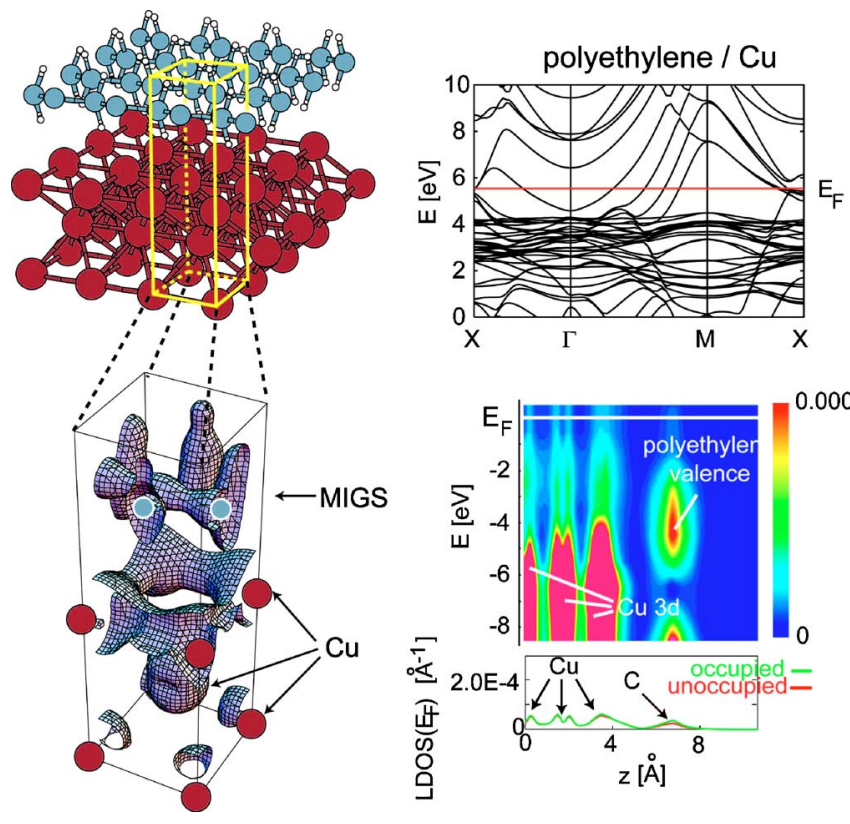

FIG. 2. (Color online) Theoretical result for the band structure of $1 \mathrm{ML}$ polyethylene/ $\mathrm{Cu}(001)$. Top left-hand inset is the atomic configuration considered here, where $\mathrm{Cu}$ is in red, $\mathrm{C}$ is in gray, and $\mathrm{H}$ is in white. Bottom left-hand side is a contour plot of the local density of states (LDOS) as a function of the growth direction $z$ and energy (color coded), while the bottom right-hand side is the LDOS at $E_{F}-0.125 \mathrm{eV}$ (occupied) and $E_{F}+0.125 \mathrm{eV}$ (unoccupied) integrated over the $x y$ plane, as a function of $z$.

dicular to the C-C-C plane, splits into two. This should be because one-half of the hydrogen atoms in the organic molecule touch the substrate in the lying-down configuration as depicted in the inset of Fig. 2, so that the $(\mathrm{C}-\mathrm{H})$ state splits due to the interaction between TTC and $\mathrm{Cu} .^{12}$

More importantly, a pronounced prepeak is seen to appear below the bulk edge onset. Because the adsorption energy for alkanes on metal surfaces is small $\left(\sim 10 \mathrm{~kJ} / \mathrm{mol} / \mathrm{CH}_{2}\right.$ chain), the chemical nature of the interface should be a typical physisorption state with a weak molecule-surface interaction, as contrasted with a chemisorption with chemical bonds formed at the interface. The prepeak should originate from the proximity to a metal rather than from chemical bonds. So we assign the prepeak as coming from the MIGS. At the present stage, we cannot completely rule out other possible origins of the prepeak such as the final-state interactions. The final state interaction between electrons and electron-vacancies can distort or produce additional peak in NEXAFS. ${ }^{13}$ However, these effects would be tiny when the interatomic interaction is weak. ${ }^{14}$ So the assignment of the prepeak to the MIGS should be reasonable.

The right panel of Fig. 1 is a blowup of the absorption edge for $1 \mathrm{ML}$ TTC/Cu(001). We can see that the prepeak is greater for a grazing x-ray incidence than for the normal incidence. This indicates that the MIGS wave functions are oriented in the surface normal direction. ${ }^{11}$

\section{AB INITIO CALCULATION}

Let us now move on to the first-principles (density functional theory) electronic-structure calculation for the hetero- 
interface to examine how the above experimental result fits the theoretical picture. Quite recently Morikawa et al. ${ }^{15}$ have performed a first principles calculation for interfaces between alkane and various metals such as $\mathrm{Cu}$, where the change in the work function and a softening of the $\mathrm{CH}$ stretching mode were studied. Here we explore the local density of states, and also predict what will happen when we replace the substrate with a ferromagnetic $d$-band metal such as $\mathrm{Ni}$, for which the spin-density functional theory is employed. The density of unoccupied states of $\mathrm{Ni}$ should be much greater than that of $\mathrm{Cu}$, since $\mathrm{Ni}$ is ferromagnetic with the major part of the minority-spin $3 d$ band sitting above the Fermi energy. So, naively, the intensity of MIGS for organic/Ni is expected to be much stronger than that for organic/Cu, which has motivated us to study $\mathrm{Ni}$.

A penalty for doing a first-principles band calculation is, given the complexity of the system, we must replace the alkane with a finite $n$ with polyethylene, assumed to be infinitely long. This reduces the size of the unit cell, enabling us to perform the spin density functional study. We have also assumed that polyethylene chains are close-packed, while an experiment ${ }^{10}$ for TTC indicates that the real packing has half this density. However, the formation of the MIGS is in general dominated basically by the band gap of the insulator and the charge transfer from the insulator to the metal (or vice versa). ${ }^{5}$ So, since the work function and the band gap of polyethylene should not drastically depend on whether the molecules are close packed or not, we can expect that the present calculation should capture essential features of the electronic structure of the interface.

We adopt the exchange-correlation functional introduced by Perdew et al. ${ }^{16}$ and employ ultrasoft pseudopotentials in separable forms. ${ }^{17,18}$ The cutoff energy of the plane-wave expansion for the wave function is taken to be $42.25 \mathrm{Ry}$. The atomic configurations (inset of Fig. 2) and the corresponding electronic ground states are obtained with the conjugate gradient scheme. ${ }^{19}$

Figure 2 shows the band structure along with the local density of states (LDOS) for polyethylene/Cu. The LDOS at $E$ is calculated as $\Sigma_{i}\left|\phi_{i}(x, y, z)\right|^{2}$ with the summation taken over the eigenstates having energies $E-0.125 \mathrm{eV}<E_{i}<E$ $+0.125 \mathrm{eV}$. The number of sampled $k$ points is eight with the Monkhorst-Pack method for the integration over the Brillouin zone, ${ }^{20}$ where the bands are fitted to sinusoidal forms and the tetrahedron method is employed. We can see in the result that LDOS at $E_{F}$ has peaks at the carbon sites, which indicates MIGS are formed at the polyethylene/ $\mathrm{Cu}$ interface. The wave function contour (inset of Fig. 2) confirms this in real space. The MIGS wave functions are seen to be oriented in the surface normal direction, which also agrees with the above experimental result.

Ni substrate: We move on to polyethylene/Ni in Fig. 3, where we must look at the majority- and minority-spin components separately, since the Ni substrate is spin polarized. We can see that the local density of states around $E_{F}$ significantly differs between the majority and minority spins, including those at the carbon sites. In the energy-resolved LDOS (color-coded panel in Fig. 3) this is seen to come from a difference in the positions of MIGS in the occupied $(E$ $\left.<E_{F}\right)$ side. To be more precise, there is a finite exchange
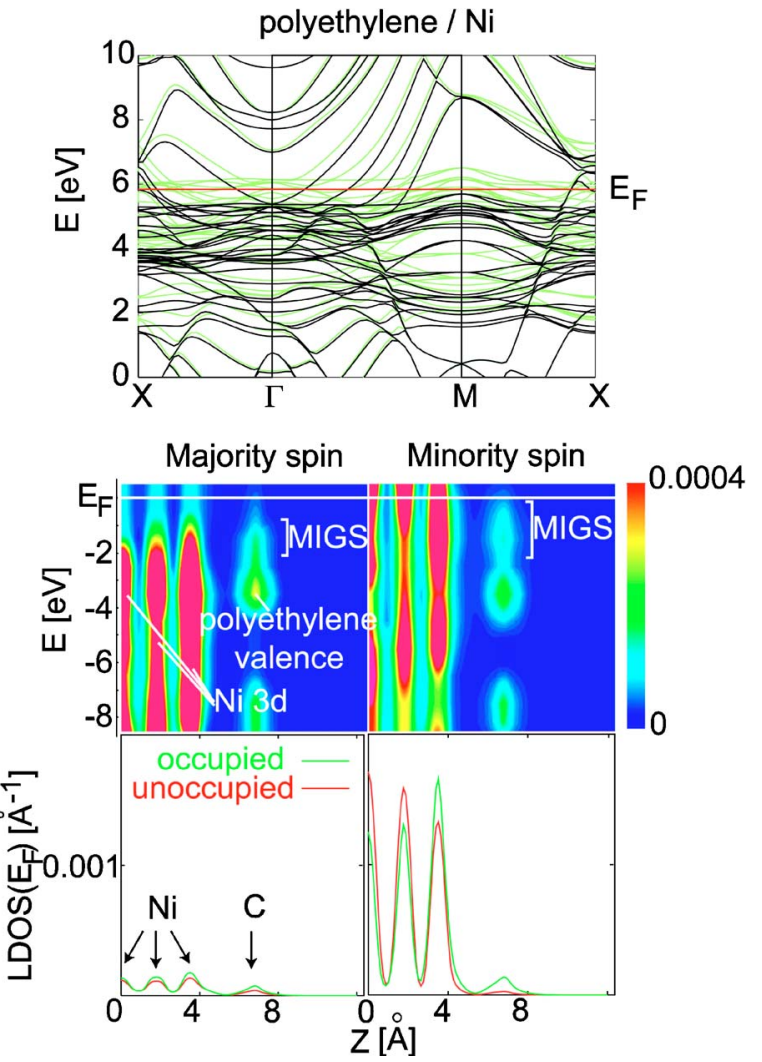

FIG. 3. (Color online) A plot similar to Fig. 2 for $1 \mathrm{ML}$ polyethylene/ $\mathrm{Ni}(001)$, where the spin density functional theory is adopted. Green (black) lines in the band structure represent the majority (minority) spin.

splitting for the MIGS in the polyethylene/Ni interface, although the splitting is smaller than the splitting within the $\mathrm{Ni}$ substrate. However, both the majority- and minority-spin MIGS lie below $E_{F}$ (with the latter lying just below $E_{F}$ ). This implies that the organic crystal, although lying on a ferromagnetic substrate, is not spin-polarized. Figure 4 compares for $\mathrm{Ni}$ and $\mathrm{Cu}$ substrates the total (i.e., sum of the majority and minority spin) LDOS (which is relevant to the NEXAFS). In accord with the above, the density of unoccupied MIGS states is similar between polyethylene/Ni and

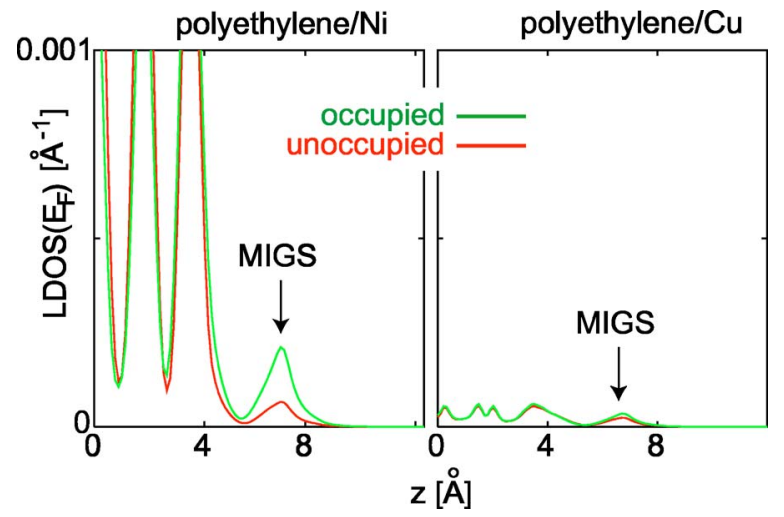

FIG. 4. (Color online) Theoretical results for the local density of occupied and unoccupied states at $E_{F}$ are compared for polyethylene/Ni and polyethylene/Cu. 


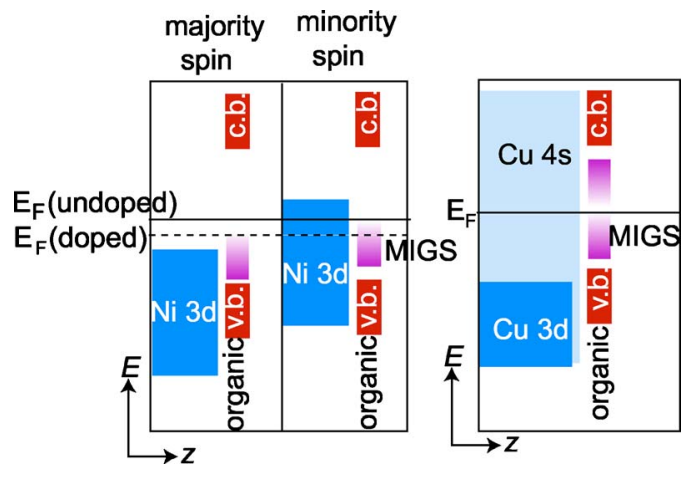

FIG. 5. (Color online) A schematic energy diagram for MIGS in organic/Ni and in organic/Cu.

polyethylene/Cu, while the density of occupied MIGS states differs between them because the $3 d$ band resides just below $E_{F}$ for Ni. The theoretical prediction agrees with our preliminary NEXAFS result, a probe for unoccupied states, for octane $\left(\mathrm{C}_{8} \mathrm{H}_{18}\right)$ on $\mathrm{Ni}(111)$ and $\mathrm{Cu}(111)$ substrates. The intensity of prepeak is similar between the two systems, with the intensity normalized by the edge jump being 0.27 for octane/ $\mathrm{Ni}(111)$ against 0.30 for octane/ $\mathrm{Cu}(111)$.

We can summarize the band scheme in Fig. 5, which schematically depicts the energy regions for MIGS in the organic/Cu and organic/Ni interfaces. The MIGS band for the majority spin in polyethylene/Ni lies below $E_{F}$, so the density of states in the unoccupied side is small, while the density of occupied states is large. By contrast, $E_{F}$ runs right through the MIGS band when the substrate is $\mathrm{Cu}$, but the density of MIGS is relatively low due to a low density of states of the $\mathrm{Cu} 4 s$ band. This results in little difference between organic/Ni and organic/ $\mathrm{Cu}$ as far as the density of unoccupied MIGS is concerned, so an experimental method that detects occupied states, such as resonant photoemission or x-ray emission spectroscopy, will probe the difference.

While the spin-unpolarized MIGS on a ferromagnetic substrate is a bit of a disappointment, the above picture naturally leads us to the following theoretical proposal. In the energy diagram in Fig. 5, we can make the MIGS spinpolarized if we can introduce carriers into MIGS by, e.g., chemical doping. Namely, while normally the majority and minority spins are (almost) fully occupied in organic/Ni, the doped carriers will be accommodated in the minority-spin band as indicated by a dashed line in Fig. 5, so that we should end up with polarized organic molecules. However, whether the argument based on the rigid-band picture is valid is subtle, which should be a future problem.

\section{CONCLUSIONS}

We have obtained a clear evidence that MIGS are formed at atomically well-defined organic-insulator/metal interfaces by using NEXAFS. Ab initio electronic structure calculation supports the existence of MIGS at the organic-insulator/ metal interface. Theoretical result indicates that the density of unoccupied MIGS states is similar between polyethylene/ $\mathrm{Ni}$ and polyethylene $/ \mathrm{Cu}$, while the density of occupied MIGS states should be different, and that a doping will make the organic molecule spin-polarized on $\mathrm{Ni}$ if an appropriate doping can be done.

\section{ACKNOWLEDGMENTS}

Calculations were performed with TAPP (Tokyo ab-initio program package), for which RA received technical advices from Y. Suwa. SR8000 in ISSP, University of Tokyo, was used for the numerical calculations. This work was supported in part by creative scientific research Grant No. 14GS0207 and a special coordination fund from the Japanese Ministry of Education.
*Present address: Department of Chemistry, Hokkaido University, Sapporo 060-0810, Japan.

†Present address: Max-Planck-Institut für Festköoperforschung, Stuttgart, Germany.

${ }^{1}$ S. Lukas, G. Witte, and Ch. Wöll, Phys. Rev. Lett. 88, 028301 (2002).

${ }^{2}$ H. Ishi, K. Sugiyama, E. Ito, and K. Seki, Adv. Mater. (Weinheim, Ger.) 11, 605 (1999).

${ }^{3}$ J. Tersoff, Phys. Rev. Lett. 52, 465 (1984).

${ }^{4}$ M. Kiguchi, R. Arita, G. Yoshikawa, Y. Tanida, M. Katayama, K. Saiki, A. Koma, and H. Aoki, Phys. Rev. Lett. 90, 196803 (2003)

${ }^{5}$ R. Arita, Y. Tanida, K. Kuroki, and H. Aoki, Phys. Rev. B 69, 115424 (2004).

${ }^{6}$ M. Kiguchi, G. Yoshikawa, S. Ikeda, and K. Saiki, Phys. Rev. B 71, 153401 (2005).

${ }^{7}$ For organic molecule/metal systems $[$ octane/Cu(110)], the interface states were found by $\mathrm{H}$. Öström, L. Triguero, M. Nyberg, H. Ogasawara, L. G. M. Pettersson, and A. Nilsson, Phys. Rev.
Lett. 91, 046102 (2003). However, ordered structures are not formed there, which cannot be described with the band picture.

${ }^{8}$ M. Kiguchi, S. Entani, K. Saiki, T. Goto, and A. Koma, Phys. Rev. B 68, 115402 (2003).

${ }^{9}$ M. Kiguchi, S. Entani, K. Saiki, and G. Yoshikawa, Appl. Phys. Lett. 84, 3444 (2004).

${ }^{10}$ Y. Hosoi, Y. Sakurai, M. Yamamoto, H. Ishii, Y. Ouchi, and K. Seki, Surf. Sci. 515, 157 (2002).

${ }^{11}$ The intensity of the NEXAFS peak is $I=\cos ^{2} \theta$, where $\theta$ is the angle between the electric vector of $\mathrm{x}$ ray and the transition moment. While the transition moment normal to the surface tends to be more strongly detected with decreasing $\theta$, the true grazing incidence is not essential for qualitatively discussing the anisotropy of MIGS. So we adopted $30^{\circ}$ as a grazing incidence angle.

${ }^{12}$ H. Kondo, F. Matsui, Y. Ehara, T. Yokoyama, and T. Ohta, Langmuir 17, 8178 (2001).

${ }^{13}$ J. L. Dehmer, A. F. Starace, U. Fano, J. Sugar, and J. W. Cooper, Phys. Rev. Lett. 26, 1521 (1971). 
${ }^{14}$ D. A. Muller, D. A. Shashkov, R. Benedek, L. H. Yang, J. Silcox, and D. N. Seidman, Phys. Rev. Lett. 80, 4741 (1998).

${ }^{15}$ Y. Morikawa, H. Ishii, and K. Seki, Phys. Rev. B 69, 041403(R) (2004).

${ }^{16}$ J. P. Perdew, K. Burke, and Y. Wang, Phys. Rev. B 54, 16533 (1996).
${ }^{17}$ D. Vanderbilt, Phys. Rev. B 41, R7892 (1990).

${ }^{18}$ K. Laasonen, A. Pasquarello, R. Car, C. Lee, and D. Vanderbilt, Phys. Rev. B 47, 10142 (1993).

${ }^{19}$ J. Yamauchi, M. Tsukada, S. Watanabe, and O. Sugino, Phys. Rev. B 54, 5586 (1996).

${ }^{20}$ H. J. Monkhorst and J. D. Pack, Phys. Rev. B 13, 5188 (1976). 